\title{
Incomplete conditionals and the syntax-pragmatics interface
}

\author{
Chi-Hé Elder, University of East Anglia \\ Eleni Savva, University of Cambridge
}

\begin{abstract}
$\underline{\text { Abstract }}$
This paper is concerned with conditional thoughts that are expressed via 'incomplete conditionals' in which an if-clause is uttered with no corresponding main clause, and yet still succeeds at communicating a fully-fledged conditional proposition. Incomplete conditionals pose a puzzle for the semantics and pragmatics of conditionals as in one respect, a condition is expressed explicitly using the canonical form 'if $p$ ', yet in another, the target of the condition is left unexpressed, requiring recourse to other linguistic or extra-linguistic information for its recovery. Taking observations from attested corpora, we explore the various ways in which the consequent of an incomplete conditional can be recovered, demonstrating that cases of incompleteness range from simple cases of ellipsis which are susceptible to a syntactic solution at one end of the continuum, to pragmatically recoverable cases at the other. This involves considering aspects of meaning arising out of the co-text, including cross-sentential anaphoric dependencies and considerations of coherence, as well as extra-linguistic context such as shared sociocultural information and world knowledge.
\end{abstract}

\section{Keywords}

Incomplete conditionals, subsentential speech, fragments, conditional thought

\section{Introduction}

It is well reported that conditionals of the form 'if $p, q$ ' can give rise to a range of nonconditional 'pragmatic' readings. For example, they can be used to highlight the relevance of $q$ as in (1), as politeness markers as in (2), or to hedge the content of $q$ as in (3).

(1) If you're looking for your keys, they're on the table.

(2) If you wouldn't mind, could you close the door?

(3) If I remember rightly, it's Tuesday today.

While in canonical cases $p$ is expected to describe a condition for the truth of $q$ (hence, 'conditional'), non-conditional meanings such as those in (1) to (3) typically arise when $p$ functions not as a condition for the truth of $q$, but as a condition for the felicity of $q$ as performing a successful speech act. The dominant view on the semantics of conditional sentences that express non-conditional thoughts makes use of Gricean reasoning: since the main meaning expressed diverges from the explicit content of what is said, the speaker must have had independent contextual reasons for using a conditional form that licenses the non-

\footnotetext{
${ }^{1}$ Authors are listed in alphabetical order.
} 
conditional reading (see Grice 1967 on the mismatch between natural language conditionals and the material conditional; see e.g. Franke 2009 on the conditional relationship between $p$ and $q$ ).

This paper is not about conditional sentences expressing non-conditional meanings. Rather, this paper is concerned with a species of conditional thought whose primary meaning is not recoverable by looking at the relationship between $p$ and $q$, precisely because at the level of the uttered sentence, there is no explicit relationship to be recovered in the first place. That is, this paper is concerned with what we call 'incomplete conditionals': conditional thoughts that are expressed via a conditional sentence fragment of the form 'if $p$ ', as in the clause marked in bold in (4) (example from the Great British International Corpus of English).

(4) (Casual conversation about common friends)

A: And Karen and Ian want to buy her half of the mortgage out, so they'll have too much mortgage.

B: Yeah... It really is...

A: I know. With Ian only a tennis coach.

B: Well even now. I mean, if he has good rates, good bank rates, and he's got a steady job...

A: That's true.

(ICE-GB, S1A-036: 035)

The defining feature of incomplete conditionals is that an if-clause is uttered with no corresponding main clause, and yet the if-clause still succeeds at communicating a fullyfledged conditional proposition.

Incomplete conditionals pose a puzzle for the semantics and pragmatics of conditionals as in one respect, a condition is expressed explicitly using the canonical form 'if $p^{\prime}$, yet in another, the target of the condition is left unexpressed, requiring recourse to other linguistic or extra-linguistic information for its recovery. To put it another way, in one respect we have a very direct relationship between the sentence form used and the intended meaning in that the use of an if-clause gives rise to a conditional reading, while in another, the gap between form and meaning couldn't be further apart as the intended meaning is left unarticulated and requires recourse to other resources to fill in that gap. So pragmatic inferencing is not required to recover a non-conditional pragmatic reading as in (1) to (3), but rather, pragmatic inferencing is required to fill in the missing content of the directly expressed conditional form in order to render a fully propositional conditional.

The phenomenon of meaningful incomplete sentential utterances in general is perhaps the most obvious manifestation of the mismatch between form and meaning that is attested in language. Assuming that the goal of a theory of communication is to reflect the ways in which people use and understand language in ordinary discourse, we view it the task of such an explanatorily adequate theory to account for this absence of a one-to-one mapping between structures and meanings. This leaves open the debate of the appropriate division of labour between semantics and pragmatics, and one of the most crucial questions that 
therefore has to be asked is whether the sentence is, as traditionally seen in formal semantic and syntactic approaches to language, appropriate as the basic unit of semantic, truthconditional analysis, or whether this assumption is too restrictive in that it leaves aspects of meaning only observable at the level of discourse to be pushed to the 'pragmatics wastebasket'. If we start with the sentence, an incomplete conditional will likely be viewed as simply that: incomplete; hence, such instantiations in natural language would remain outside the remit of semantic theory. The arguments in favour of utterance-based approaches to the study of truth-conditional semantics ${ }^{2}$ are well attested (e.g. Carston 2002, Recanati 2010, Jaszczolt 2016 among many others), and this paper takes the case of incomplete conditionals as evidence in favour of the contextualist view that utterance-based semantics fares better at generating intuitive truth conditions than sentence-based semantics. Indeed, we demonstrate that intuitively truth-conditional content can be recoverable from an implicitly communicated consequent on the basis of pragmatic considerations, even in the absence of any explicitly uttered linguistic material. We thus take the case of incomplete conditionals as a fruitful case study in showing that syntactically-driven accounts of meaning are too restrictive to do justice to the empirical facts.

At this point, a disclaimer is in order. We rely on the intuition that speakers are able to - and do - assign truth values to natural language conditionals as they are used in context, and hence that conditionals are amenable to a truth-conditional treatment. On this intuitive basis, we resist the view that truth conditions are a 'philosophers' fiction', and instead aim to provide an explanatorily adequate view of the truth conditions of natural language conditionals as they are used and understood in ordinary discourse. ${ }^{3}$ For the sake of argument, we follow Ramsey's (1929) intuition that people make judgements about $q$ assuming $p$ as given, and as such assume a Stalnakerian view on the truth conditions of conditional utterances, where an uttered sentence of the form 'if $p, q$ ' is true if and only if in the closest possible world where $p$ is true, $q$ is also true (Stalnaker 1975). But note that the goal of this paper is not to comment on the truth conditions of conditional propositions, but rather to show that interlocutors rely on much more than the syntactic structure to recover the missing content of incomplete conditionals such that the relevant truth conditions can be applied at the propositional level.

To this end, in this paper we explore the various ways in which the consequent of an incomplete conditional can be recovered. In doing so, we demonstrate that incompleteness is not only a pragmatic phenomenon, but can potentially be associated with different levels of representation, from syntactic solutions at one end of the continuum, to almost entirely pragmatically recoverable cases at the other in which the linguistic form in isolation is not sufficient to generate a completion and requires interaction with extra-linguistic information. Based on a classification of incomplete utterances (Savva 2017), at one end of the spectrum

\footnotetext{
${ }^{2}$ Or 'truth-conditional pragmatics', as labelled by Recanati (e.g. 2010), but this is a matter of terminological preference as opposed to theoretical commitment.

${ }^{3}$ As we are working within the framework of truth-conditional semantics, probabilistic approaches to conditionals are not discussed here, but the interested reader is directed to seminal works such as Adams (1975) and Edgington (1986).
} 
we find simple cases of ellipsis giving rise to syntactic incompleteness where the recoverable completion has a determinate syntactic structure (cf. Merchant 2004). In the middle, we move to consider issues of discourse coherence, where the issue of how determinate the syntactic structure of the recovered completion is becomes fuzzier, leading us into the debate of whether the non-expressed aspects of truth-conditional content correspond to variables in the logical form (cf. Stanley 2000) or whether they are implicitly expressed (cf. Bach 2007). For the sake of psychological plausibility, we favour the latter approach, which moves us to the other end of the spectrum, at which there are cases for which the recoverability of the communicated thought depends on an interaction between linguistic and extra-linguistic information, and the corresponding proposition lacks a determinate syntactic form altogether (cf. Stainton 2006). Overall, we suggest that incomplete conditionals pose no problem to a theory of conditionals, or even semantic theory at large, when truth conditions are allowed to operate on a unit of a wider scope that can encompass information provided by the syntactic structure, inter-sentential relations, as well as extra-linguistic pragmatic phenomena. This involves considering aspects of meaning arising out of the co-text, including cross-sentential anaphoric dependencies and considerations of coherence, as well as extra-linguistic context such as shared sociocultural information and world knowledge.

The structure of the paper is as follows. We begin by providing a definition of conditional thought that allows us to include incomplete conditionals in its scope, facilitated by viewing the concept of conditionality at the level of thought. We then consider the ways in which incompleteness is attested in natural language. Based on a corpus of incomplete conditionals compiled from the Great British component of the International Corpus of English (ICE-GB), we identify different types of instances of incomplete conditionals according to whether the recovery of their completion relies more heavily on syntactic information or on pragmatic considerations. Ultimately, we argue that it is only by allowing the truth-conditional unit to incorporate syntactic and pragmatic information alike that we can derive propositional meanings from incomplete conditional structures that reflects the ways in which interlocutors use such structures in natural language.

\section{Pragmatic conditionals at the level of thought}

The study of conditionals has focussed in the main on utterances of the form 'if $p, q$ ', both in terms of the truth conditions (or lack thereof) of their corresponding propositions, and on the range of pragmatic meanings they can be used to express. However, comparatively little research has questioned what happens when the uttered conditional pertains only to a sentence fragment. An exception to this is 'isolated if-clauses' used to express 'polite directives' (e.g. Ford and Thompson 1986), as in (5).

(5) Now if you'd like to put on your helmet. (ICE-GB, S2A-054: 063)

As acknowledged by Stirling (1999), such isolated if-clauses are typically used to issue requests, and any reconstructed main clause is likely to pertain to the speaker's wishes (e.g. 
'that'd be great') as opposed to the hearer's desires (as indicated by 'if you'd like to' in this example). Stirling also identifies a similar construction that uses isolated if-clauses to express 'optatives', expressing a (often counterfactual) wish of the speaker, as in (6).

(6) If only Miss Hawkins would get a job... (FLA p.195, from Stirling 1999: 286)

Quirk et al (1985: 841) categorise these optatives as 'irregular sentences', commenting on their exclamatory function: "the omission of the matrix clause [...] being mimetic of speechless amazement”. Again, any main clause that could be postulated is likely to express a positive outcome from the speaker's point of view.

While Stirling (1999) notes that isolated if-clauses of both types can perform similar functions to those of fully-fledged conditional clauses with overtly expressed main clauses, she argues that the construction is undergoing a process of grammaticalization, with the subordinate clause being promoted to the status of main clause. Evans (2007) labels this process 'insubordination', with supporting examples of isolated if-clauses from a range of languages, including from French, Dutch, Spanish, Basque, and Japanese. So, the same arguments that license such constructions as having undergone morphosyntactic change also drive our motivation to exclude them from our analysis of incomplete conditionals. That is, such isolated if-clauses are of lesser interest to this paper insofar as, from a semantic point of view, arguably no main clause is required for their successful transmission of meaning. Instead, we assume that these conventional uses of if-clauses likely license generic or recurring main clauses, where there is no 'fresh' completion to be recovered. So, irrespective of whether there is a determinate main clause to be recovered, one could plausibly argue that the primary function of any inferred main clause would not be to communicate content, but to support the speech act (e.g. of a polite directive) generated by the if-clause alone (cf. Vallauri 2004), and hence are not intended to communicate conditional thoughts.

The cases that are of greater interest to us are non-grammaticalized instances of isolated if-clauses which arise when the conditional clause alone is self-evident as an expression of the full content of the conditional thought. In such cases, a main clause is not necessarily required to be overtly expressed for the expression of a proposition, and in some cases, may even be redundant and costly to produce (cf. Elder and Beaver 2017). However, the implicitly communicated consequent of the conditional thought is required insofar as it contributes independent, context-specific content. This is in contrast to Stirling's (1999) study which explicitly excluded conditionals with a covert, contextually supplied consequent, focussing instead on isolated if-clauses used as standalone directives or optatives.

From a database of if-conditionals obtained from the International Corpus of English (GB) compiled by one of the authors, we identified 76 instances of incomplete conditionals. ${ }^{4}$ Of these, 47 (62\%) were of the conventionalised type, either performing the role of a polite directive or an idiomatic expression. Given their conventionalization, it is perhaps not too

\footnotetext{
${ }^{4}$ The ICE-GB comprises 300 2000-word samples collected over a range of discourse contexts. As detailed in (Elder 2014), a lexical search for 'if' yields 2068 tokens. Removing indirect questions (where 'if' could be substituted for 'whether'), 'as if', and unintelligible tokens, 1702 tokens remain, of which 76 constitute $4 \%$.
} 
surprising that these constituted the majority of the cases of incomplete conditionals, but, as already discussed, since such cases do not require any filling in of a consequent to obtain the main intended reading, we leave these cases out of our analysis. Rather, we focus our attention on the remaining 29 (38\%) cases: conditionals which are incomplete in their surface form, but require pragmatic inferencing for their completion. ${ }^{5}$ In this sense we can draw parallels between conventionalized versus non-conventionalized incomplete conditionals and Grice's (1989) familiar Generalized Conversational Implicature (GCI) and Particularized Conversational Implicature (PCI) distinction: the intended meanings of the former are recoverable via an automatic inference irrespective of the context in which they occur, while for the latter, the completion of the conditional is both necessary for conveying a complete thought, and, moreover, is only available in its specific context of utterance.

The view that conditional propositions have to be construed at the level of thought is compatible with Elder and Jaszczolt's (2016) 'pragmatic category of conditionals', in which conditionality is defined so as to encompass the variety of ways that conditional thoughts can be expressed in natural language: both with and without 'if', and in both English and crosslinguistically. While they acknowledge incomplete conditionals of the conventional type, we add to the list our non-conventional incomplete conditionals, adding further support to the view that conditionality has to be defined pragmatically at the level of thought. We thus follow suit in conceptualising conditionals as adhering to their criterion that $p$ provides a restriction on $q .{ }^{6}$ Specifically, the reference to $q$ in the criterion for conditionality highlights conditionals as comprising two-part thoughts. However, in the case of incomplete conditionals, the two-part conditional thought is only partially expressed: while the linguistic input of the 'if $p$ ' form signals what the structure of the complete thought will look like, it is necessary to fill in the consequent to obtain a full conditional proposition. For example, in the case of (4), repeated below, the if-clause does not make sense in isolation.

(4) (Casual conversation about common friends)

A: And Karen and Ian want to buy her half of the mortgage out, so they'll have too much mortgage.

B: Yeah... It really is...

A: I know. With Ian only a tennis coach.

B: Well even now. I mean, if he has good rates, good bank rates, and he's got a steady job...

A: That's true.

(ICE-GB, S1A-036: 035)

It is only in the context of utterance that the if-clause can interact with the prior discourse, which makes available a completion along the lines of 'he could afford to pay the mortgage' and hence gives rise to a fully-fledged proposition (cf. Section 4.3).

\footnotetext{
${ }^{5}$ The quantitative information is provided to draw attention to the frequency of the phenomenon; in the remainder of the paper we simply use the instances found in the corpus as examples for discussion.

${ }^{6}$ Note that this criterion is a necessary condition for expressing a conditional proposition but not a sufficient one. See Elder and Jaszczolt (2016) for discussion.
} 
We assume that incomplete conditionals have the truth conditions of other regular, hypothetical conditionals. To repeat, we subscribe to the truth conditions of conditionals as given to us by Stalnaker (1975): a conditional 'if $p, q$ ' is true if and only if in the closest possible world where $p$ is true, $q$ is true. However, in the absence of complete syntactic structures, rather than applying these truth conditions to conditional sentences, we apply them at the level of thought. Again, it is only once the consequent is filled in that we obtain a conditional proposition, and hence that truth conditions can successfully be applied. However, the question that remains is how to recover the unpronounced consequent. To inform our solution, we turn to the extant literature on subsentential speech to cast light on how we can derive the required truth conditions of these incomplete conditionals.

\section{The role of syntax versus pragmatics in recovering complete thoughts}

Incomplete conditionals are a subclass of the phenomenon of 'subsentential speech' or 'fragments'; that is, linguistic structures that are incomplete from a syntactic point of view but can convey complete thoughts when uttered in context. We use the term 'subsentential' to refer to any structure that is not syntactically complete, i.e. that is not a full complementiser phrase $^{7}$ in the standard terminology of Generative Syntax. In English, these are usually clauses that do not contain a finite verb. In the case of conditionals of the form 'if $p$ ', syntactic incompleteness is rather straightforward to spot insofar as such constructions lack an uttered main clause but can nevertheless be used to communicate a fully-fledged conditional thought. And, taking it as given that interlocutors do succeed in communicating complete propositions with the use of grammatically incomplete sentences, it is necessary that our semantics allows us to bridge the gap between the incomplete syntactic form and the communicated thought.

Regarding how that gap might be bridged, we turn to extant accounts of the broader phenomenon of subsentential speech, which give us two main options. On the one hand, we may assume that the understood completion is already present in the structure of the uttered sentence, essentially viewing the completion as a case of syntactic ellipsis (along the lines of e.g. Stanley 2000, 2007; Merchant 2004, 2010; Ludlow 2005; Martí 2015). Accounts of syntactic ellipsis take the 'missing' part of a seeming subsentence to be phonologically unarticulated yet present in the syntactic structure, allowing the meaning to be computed compositionally (see van Craenenbroeck and Merchant 2013 for definitions and examples of different types of ellipsis). Alternatively, we might view the communicated completion as pragmatically recoverable based on contextual information (following e.g. Barton 1990; Stainton 2006; Hall 2009). ${ }^{8}$ In this paper, we follow Savva (2017) and take a middle ground, arguing that subsentential speech is, in fact, a diverse phenomenon that cannot be accounted for in its totality by either one of these two extreme positions. We maintain that some instances of subsentential speech lend themselves most naturally to an account of syntactic

\footnotetext{
${ }^{7}$ Nowadays, a complementiser phrase is taken to correspond to a syntactically complete sentence in Generative Syntax, but the term IP (inflection phrase) is also sometimes used (see Cinque and Rizzi 2010 for a discussion). ${ }^{8}$ See Bezuidenhout (2006) for an overview of this debate.
} 
ellipsis; however, rather than overextend that account beyond what is justifiable, we contend that instances in which pragmatics plays a significant role in recovering the completion must also be included in our semantic theory. We thus take incomplete conditionals as a case study that exemplifies the diversity involved in the phenomenon of subsentential speech and show that different instances draw on syntactic and pragmatic features to different degrees. Before presenting the range of ways in which incomplete conditionals can be used to express complete thoughts, and hence the ways in which those complete thoughts can be recovered, in this section we first outline some of the problems with attempts to overextend accounts of syntactic ellipsis to all cases of subsentential speech, highlighting the importance of admitting pragmatic considerations into a truth-conditional semantic theory.

The view that seemingly subsentential utterances are, in fact, syntactically complete is made possible if one postulates either unpronounced structures (e.g. Merchant 2004, Martí 2015 ) or hidden slots (e.g. Stanley 2000, 2007) in the logical form of the uttered sentence, rendering the utterance syntactically complete in its underlying logical form. For example, on Merchant's (2004) account, cases of subsentential speech fall into two categories: examples such as (7) require postulating the phonologically unpronounced verb phrase 'do it' in the logical form of the uttered sentence, while examples like (8) require postulating the determiner phrase 'this is' (examples from Savva 2017).

(7) [I am offered a second slice of cake.]

\section{I really shouldn't [do it].}

(8) [At a house gathering, I am talking to my friend when a man walks in. We both notice him.]

\section{[This is] my brother.}

These postulations turn (7) and (8) into fully sentential structures, after which they become as context-dependent as any (fully sentential) utterance requiring reference resolution.

Merchant's account diverges from a commonly acknowledged feature of syntactic ellipsis, namely that an ellipsis site needs to be licensed by an appropriate linguistic antecedent. Indeed, one of the main arguments for taking a pragmatic - as opposed to syntactic - approach to subsentential speech is that cases of ellipsis cannot appear in discourse-initial position, thus rendering subsentential speech proper a different phenomenon from that of syntactic ellipsis (see, for example, Yanofsky 1978; Elugardo and Stainton 2005; Stainton 2006). However, by postulating one of these two very specific constituents, Merchant (2004) sidesteps this requirement and allows that syntactic ellipsis does not require a linguistic antecedent as long as there is a well-defined extra-linguistic context which can serve as an appropriate antecedent. He thereby argues that seemingly discourse-initial 
subsentences, such as those in (7) and (8), are not, in fact, discourse-initial, and hence can be subsumed as cases of syntactic ellipsis. ${ }^{9}$

Stanley (2000: 404-405) makes a similar move, arguing that the extra-linguistic context can give rise to a 'linguistic' antecedent without it having to be explicitly uttered; for example, a questioning gaze from a friend could be interpreted as 'Who is that man?', thus providing an appropriate linguistic antecedent to the utterance in (8). Now, we view nothing wrong with considering a gesture or other salient extra-linguistic information part of the discourse; in fact, we expect that subsentences are very often used in response to nonlinguistic communicative acts, and for those subsentences to achieve their communicative goals, extra-linguistic material needs to be well-defined. However, as soon as the discourseinitial position is offered to extra-linguistic information, the distinction between linguistic and non-linguistic factors becomes blurred. It seems that in making this move, the effort to maintain subsentential speech as a purely syntactic phenomenon without contextual intrusion fails. While a questioning gaze can "raise a linguistic expression to salience" (Stanley 2000: 404), the gaze itself does not have syntactic structure; it is due to pragmatic factors that it becomes interpretable, and it is thus not clear how its interpretation is any more linguistically constrained than interpreting 'kicks under the table and taps on the shoulder' - gestures that Stanley (2000: 396) specifically considers irrelevant to linguistic theory. Rather, we take these accounts that extend ellipsis-style arguments to more 'pragmatic' instances as evidence that subsentential speech is, indeed, more dependent on context and pragmatic inference than syntactic ellipsis is, and that an explanatorily adequate account of subsentential speech has to allow for an interaction of linguistic and extra-linguistic information. Indeed, if we simply accept appearances and assume that there is no linguistic term present to be filled in by contextual information, then subsentential utterances become similar to other phenomena that require top-down contextual enrichment (e.g. Carston 2002; Recanati 2004, 2010) in order to make sense of what the speaker said.

If the goal is to determine fully sentential structures from subsentential ones, once we have found a suitable antecedent from which to recover the incomplete structure, the problem ends there. However, if the goal is to capture the intuitive truth-conditional meaning, Merchant's and Stanley's solutions remain problematic, as completing an utterance at the structural level will only take us so far. As Bach $(1994,1999,2007)$ has repeatedly argued, syntactically complete sentences often fail to express complete propositions; while (9) and (10) are perfectly complete sentences, unless the questions in parentheses are contextually resolved, the sentences are not truth-evaluable (examples adapted from Bach 1994: 285).

\footnotetext{
${ }^{9}$ It is not actually difficult to find subsentential utterances for which a postulation of [do it]/[this is] is inappropriate, as exemplified below, and hence Merchant's (2004) account does not explain the phenomenon in its totality (see also Stainton 2006 on this point).
}

[A and B are at a student-committee meeting talking about possible party activities.] A: We want something that's going to, sort of, like, instil a little bit of fear at this point. B: Cartwheels. (ICE-GB, S1B-079: 223) 
(9) Anna is ready. (For what?)

(10) Peter has finished. (What?)

To get to this intuitive content, Merchant's and Stanley's accounts would need to be supplemented by a pragmatic theory that can explain how a complete sentence turns into a complete thought that is communicated in context. ${ }^{10}$

A further feature that differentiates syntactic ellipsis from non-elliptical subsentential speech is that in cases of the former, there is a determinate syntactic completion, as in (11), whereas in cases of subsentential speech there is more than one possible completion, as in (12) (examples from Savva 2017).

A: Who wants the last piece of cake?

B: I do [want the last piece of cake].

(12) [Anna and George are playing Scrabble. George places a word on the board. Anna, who is responsible for keeping the score, sees it and grabs her pen.]

\section{Twenty-five.}

(12a) Your word is worth twenty-five points.

(12b) I'm writing down twenty-five points for you.

(12c) You get twenty-five points in this round.

In (11), the completion of the syntactic form is unique and has to be 'copy-pasted', so to speak, from the immediately preceding discourse. By contrast, (12) could be completed by a number of possible candidates, such as any of those in (12a)-(12c). But crucially, note that in any given context, the available completions are interchangeable to the extent that - in the case of (12) - the proposition that the word on the board is equivalent to twenty-five Scrabble points is equally communicated by them all.

The notion of 'indeterminacy of form' is employed by proponents of the syntactic approach to argue that subsentences do not correspond to propositional messages unless there is determinate 'content' to be recovered (Stanley 2000: 407-408). However, what we take this distinction to show is that, once again, subsentential speech is simply less constrained by the linguistic system than ellipsis is. What matters for communication is the transmission of the speaker's intended meaning, and not the recovery of a unique syntactic completion. In fact, building on Bach's (1994) examples (9) and (10) above, indeterminacy of form is often involved to some extent in undeniably propositionally complete utterances, such as those with definite descriptions, as in (13), which could communicate any of (13a)-(13c) and many more:

(13) The book is on the table.

\footnotetext{
${ }^{10}$ For a more detailed discussion of problems with Stanley’s (2000, 2007) indexicalism, see Clapp (2012).
} 
(13a) The book The Unbearable Lightness of Being is on the table.

(13b) The book I am reading at the moment is on the table.

(13c) The book that you asked to borrow is on the table.

We thus embrace Neale's $(1990,2004)$ view that while the logical form of definite descriptions supplies the information that there is a unique entity, the referent is only fixed through an interaction between the linguistic form and the context of utterance. Equally, while there may be many ways to describe that referent and hence syntactically complete the proposition (as in 13a-13c), it does not matter which completion (s)he entertains as long as the hearer recovers the reference to the relevant book. So, indeterminacy of form is not problematic in the search for the communicated proposition as even syntactically complete sentences require an interaction with a context of utterance to determine a unique proposition.

As such, we reject indeterminacy of form as a criterion for distinguishing utterances that are not propositional from those that are (see also Clapp 2005: 120 on this point). Rather, what this criterion simply shows is that there is a difference between standard cases of syntactic ellipsis and non-elliptical cases of subsentential speech. Because ellipsis depends entirely on antecedent linguistic material for its recovery, it is possible to find a completion which is determinate in form. But because the completion of subsentential speech depends on the extra-linguistic context, and because this context does not contribute syntactic structures, it is likely that the recoverable completion will be syntactically indeterminate. And, as our analysis of incomplete conditionals in the following section demonstrates, there are cases of subsentential speech that straddle the boundary between linguistically mandated completions and pragmatically recoverable ones. Rather than separating out syntactic ellipsis as a distinct phenomenon that requires a unique analysis, we view syntactic ellipsis as a subclass of the broader phenomenon of subsentential speech, whose instances lie on a continuum that primarily utilise syntax for their completion at one end, to those that require considerable extra-linguistic pragmatic information at the other.

\section{Recovering conditional thoughts from incomplete conditionals: A continuum}

The extent to which pragmatic inferencing is called upon to recover a conditional thought for an incomplete conditional depends on the extent to which the thought can be said to be expressed explicitly. On the one hand, a conditional thought can be said to be expressed explicitly insofar as the default conditional marker 'if' is used to introduce the thought. But when the antecedent introduced using 'if' lacks its corresponding consequent, a high degree of pragmatic inferencing is potentially required to recover the conditional thought in its entirety. We thus move to consider cases of naturally occurring incomplete conditionals of the form 'if $p$ ' obtained from the ICE-GB. As it will transpire, we find that the range of ways in which incomplete conditionals can be completed such that they convey complete thoughts is diverse, and, therefore, we motivate adopting a semantic account that can take this diversity into consideration. In this section, following the taxonomy of subsentential speech offered in Savva (2017) we present a classification of incomplete conditionals whose 
instances lie on a continuum that ranges from cases susceptible to a syntactic solution to cases that heavily rely on pragmatic inference. Where each case lies on this continuum depends on both the degree of input that syntax and pragmatics contribute to recovering the completion, as well as how syntactically determinate that completion is. We employ the concept of a continuum in order to highlight that all cases on it depend on both syntactic and pragmatic information albeit to different extents, and that the difference between the classes we propose below is one of degree. With this in mind, it should be noted that the idea of a continuum is compatible with much more gradience than what might be gleaned from the classification presented in the following sub-sections which is aimed to illustrate the diversity involved in incomplete conditionals without necessarily exhausting it.

A disclaimer is necessary at this point. It may seem that syntactic and pragmatic solutions do not occupy the same level of representation, insofar as the former explain how syntactic structures are generated, while the latter explain how interpretations arise. However, from a processing point of view, the two projects need not be dissociated, insofar as speakers' linguistic competences, including their subconscious knowledge of grammatical rules, can be seen as a subpart of the totality of information that contributes to the interpretation of subsentential speech. In fact, although syntactic theory need not deal with processing matters, certain syntactic approaches explicitly view their assumptions about parsing on the basis of grammatical rules as a reflection of the human sentence processing mechanism (see, for example, Hale 2011). Following this view, we see no problem in placing syntactic and pragmatic solutions on the same continuum.

\section{$\underline{4.1 \text { Incomplete conditionals as cases of syntactic ellipsis }}$}

At the most syntax-oriented end of the continuum, we have cases of subsentential speech that can be labelled as ellipsis, insofar as their completion is dependent on finding a suitable linguistic antecedent available in the immediately preceding discourse and that can be 'copypasted' from there. As the antecedent is overtly linguistic, the completion also has a unique and, therefore, determinate - syntactic structure. In the case of incomplete conditionals, the consequent is most likely to be recovered from the content of another interlocutor's question, as in (14) and (15):

(14) A: Can I say acey-pacey?

B: If you so desire [you can say acey-pacey].

(ICE-GB, S1A-068: 009)

(15) A: It's obligatory, is it, to have something in a company report?

B: If you've got more than a hundred in the workforce [it's obligatory to have something in a company report].

(ICE-GB, S1B-062: 138) 
In (14), the consequent of the incomplete conditional in B's response can be found in A's question; if we 'copy-paste' it, we retrieve the complete conditional 'If you so desire, you can say acey-pacey'. Similarly in (15), the consequent of the conditional in B's response is, again, found in A's question, so that the complete proposition expressed by B's utterance corresponds to 'If you've got more than a hundred in the workforce, it's obligatory to have something in a company report'. Importantly, there are no other salient completions available that can constitute consequents for these incomplete conditionals, and the ellipsis site is simply filled in by considering the linguistic content of the previous utterance. ${ }^{11}$

Note that from a processing point of view, choosing suitable constituents from the preceding discourse may still require some pragmatic inference. However, this assumption is compatible with placing all types of incomplete conditionals on a unified continuum ranging from syntax to pragmatics, because what differs in each category is the degree to which syntax and pragmatics contribute to generating a completion. Indeed, even in these cases of syntactic ellipsis, completions are not completely devoid of extra-linguistic consideration due to the fact that a conditional structure is used in response to a question, and hence in each case there is a covert affirmative answer to be recovered. Given the asking of a question, the immediately next turn is expected to constitute an answer to that question. As polar yes-no questions, there are only two salient possible responses, the preferred response being the affirmative (Levinson 1983: 336). As noted in Section 2, one role of the antecedent of a conditional is to set up a condition for the content of the consequent, so it necessarily restricts the situations in which that consequent is deemed true or acceptable. In the case of (14), the if-clause puts a condition of the hearer's future action, playing the further pragmatic role of hedging the act of permission implicitly communicated. By contrast, in (15), the if-clause provides a condition on a company's obligation, thus providing the specific situation in which the consequent is expected to hold. Overall, we view it plausible to assume that syntactic ellipsis will always be resolved when there is an available linguistic antecedent on the basis that drawing on salient linguistic information is less cognitively demanding than making pragmatic inferences. ${ }^{12}$

Next, still at the syntactic end of the continuum, there are cases in which the consequent can, once again, be found in a determinate form in the preceding discourse, but not necessarily in the immediately preceding utterance. Take (16).
A: Are you actually going to bother getting a job?
B: Well, not for the next two weeks probably. I mean, I'll ask in some places in Bromley. But, I mean, if I'm going away in two weeks...

(ICE-GB, S1A-093: 023)

In (16), the consequent of the incomplete conditional in B's response can be assembled by putting together constituents from the preceding discourse, resulting in the complete

\footnotetext{
${ }^{11}$ For an example of an ellipsis account of short answers, see Merchant (2006).

${ }^{12}$ Although we leave it to future empirical research to test this point.
} 
conditional thought, 'If I'm going away in two weeks, I am not going to bother getting a job'. Note here that, again, the confirmation of B's inquiry is implicitly recovered in the consequent of the conditional. In this case, the content of the if-clause is assumed to be true, which in turn sets up the inferential basis for recovering the consequent and acting as an explanation for why the speaker won't bother getting a job. However, the case of (16) is slightly different to that of (17).
A: Is that an irritation when you have a vague feeling you've lent a book to somebody and you can't quite figure it out...it's not there?
B: If it's a paperback, no. If it's a hardback...

(ICE-GB, S1A-013: 095)

Here, the consequent of the incomplete conditional is recoverable from the previous question in the previous turn, giving us 'if it's a hardback, it's an irritation'. ${ }^{13}$ Again, it is the positive response to the question that is implicitly recovered, but rather than the if-clause setting up the inferential grounds to draw a conclusion, the if-clause provides the situation in which the consequent is true: in the situation where the book that has been lent is a hardback, it is the case that it is an irritation. The inference that the elided consequent is taken as an affirmative response to the previous question is also facilitated by the cue that the if-clause is set up in juxtaposition with the previous negative case.

Since a unique consequent is salient for all examples (14)-(17), we view these as cases of syntactic ellipsis. ${ }^{14}$ But in all of these examples, the recovered consequent serves as a response to a question, so some degree of pragmatic inferencing is required to recover whether the response is positive or negative. The difference between the cases lies in how much processing is required. We conjecture that from a processing point of view, slightly more pragmatic effort is required to choose a suitable consequent for (16) and (17) as it has to be selected from among other material in the available discourse. For this reason, we call the latter cases instances of 'co-text ellipsis' and we place them one step closer to the pragmatic end of the continuum. However, the category of co-text ellipsis could potentially be dispensed with if one adopts a dynamic framework in which syntactic representations are constructed incrementally (e.g. Cann et al. 2005). In such a case, our two subclasses of ellipsis would merge; we remain agnostic on this issue as it does not affect our argument that

\footnotetext{
${ }^{13}$ Note that, in line with contextualist assumptions, the antecedent also needs fleshing out to 'if the book that I've lent to somebody is a hardback ...', where the linguistic information in the immediately preceding turn supplies the reference for 'it'. We assume that the prior discourse would also provide a referent for 'it' in the consequent. Thank you to anonymous reviewers for pointing this out.

${ }^{14}$ Of course, there could be cases in which the recoverable consequent is highly constrained even without an explicit mention of it in the preceding discourse. However, in such cases we expect that the constrained consequent could be expressed with more than one syntactic structure; thus, such cases would not be as convincing as examples of syntactic ellipsis. Studies of priming effects support this view: speakers are likely to fill in an ellipsis site with the exact syntactic structure as that present in the immediate co-text (see e.g. Xiang et al. 2014). In other words, it is only when the consequent has been made explicit in the preceding discourse that its syntactic structure is expected to be binding.
} 
the degree to which the recoverable meaning of incomplete conditionals relies on syntactic or contextual information varies across cases on the continuum.

\section{$\underline{4.2 \text { Incomplete conditionals and discourse relations }}$}

Moving along the continuum, we have cases for which there is no available determinate syntactic structure that can play the role of the consequent of the incomplete conditional. Nevertheless, in these cases there is a recoverable consequent that arises out of considerations of links of discourse coherence. Theories of coherence (e.g. Kehler 2002; Asher and Lascarides 2003) are based on the idea that when we comprehend a discourse, we do not interpret each utterance in isolation, but we attempt to recover links that relate the utterances to one another. As a discourse unfolds, each new proposition added is assumed to be linked to the previous one(s) in a logical way; this logical link must be recoverable for the discourse to be interpretable effortlessly. ${ }^{15}$

Coherence models go beyond syntactic considerations given that the relations in question are not normally traced to elements in the structure of individual sentences; yet at the same time, these links are not entirely left to top-down pragmatic processes, because the coherence relations between propositions are arguably calculable on the basis of systematic observation of how chunks of naturally occurring discourse are organised. Moreover, such links are confined to a relatively small number of coherence relations and so they seem much more constrained than top-down pragmatic inferences that rely entirely on speakers' intentions (Asher and Lascarides 2003: 31). This is why we place cases for which the completion can arise on the basis of such coherence links in the middle of the continuum between the syntactic and pragmatic ends. That is, for cases in this category, the discourse will not provide a consequent in a determinate syntactic form, but will guide the recovery of the appropriate completion through retrievable links of coherence between propositions, as in (18) and (19).

(18) [Casual conversation about a trip to France]

B: I'm only covered to drive it in emergencies out there.
A: Yes.
$\mathrm{B}$ : If his hip gives way.

(ICE-GB, S1A-009: 228)

(19) [Casual conversation about going to the pool]

A: Have you taken any advice about protecting your leg? It would be the scars against sunburn. Using a stronger...

B: Just to wear sun block but it doesn't actually work. It's the actual heat that affects

it. If I get very very hot and the sun's directly on it.

\footnotetext{
${ }^{15}$ Examples of such common relations are Elaboration, Narration, Result, Explanation, Parallel, Correction, Contrast, Alternation, Consequence, Background, Evidence (see, for example, Asher and Lascarides 2003).
} 
In (18), the incomplete conditional arguably communicates a consequent along the lines of 'if his hip gives way I'm covered to drive', rendering the role of the if-clause to provide an example of the phenomenon previously introduced: in this case, the circumstances under which the speaker is covered to drive. Note that while we do obtain a salient consequent, it is recovered through the connection between the antecedent and propositions expressed in the preceding discourse, and as such is not expected to constitute a unique consequent; other consequents such as 'I'll be able to drive' are also possible. Similarly, in (19), the recoverable consequent for the incomplete conditional is, arguably, 'if I get very very hot and the sun's directly on it, my leg gets affected', where again, the antecedent is an example of when the speaker's leg is affected. Thus, in both cases, the incomplete conditional is connected with its preceding discourse with the coherence link of elaboration (or exemplification). The unpronounced but retrievable consequent of the conditional makes explicit this coherence link.

Note that it may seem that the completion of the conditional we have offered for these cases does not quite get at the reading we want. Given the criterion for conditionals given in Section 2, namely that $p$ restricts the situations where $q$ obtains, it may seem that for (18), the condition 'his hip gives way' is the (unique) situation that restricts 'I'm covered to drive'. However, this is clearly too restrictive; the communicated content is intuitively something more like, 'his hip giving way is an example of when I'm covered to drive'. Overriding the logical form of the utterance in this way to give a non-conditional proposition arguably makes clearer the coherence link of exemplification. However, simply completing the conditional to 'if his hip gives way, I am covered to drive' as we do is not a problem as far as the pragmatics of conditionals goes. The reason for this apparent mismatch is that the resulting conditional proposition does not allow for a bi-conditional 'perfected' reading of the form 'if and only if his hip gives way, I am covered to drive', as $p$ simply describes one (of several potential ways) in which $q$ can obtain.

Our view on the pragmatics of conditionals thus runs counter to the dominant neoGricean approach to Conditional Perfection (CP), where CP is analysed as a scalar GCI (e.g. Atlas and Levinson 1981, van der Auwera 1997, Horn 2000): the view that if the speaker did not intend $p$ to be a necessary condition for $q$, he should either have provided the other sufficient conditions for $q$; or in the case where $q$ is not conditional on any antecedent, asserted $q$ outright. But implicit in this GCI view is the assumption that $\mathrm{CP}$ is a stereotypical inference arising from the word 'if', and it is this assumption that we contest. Indeed, scholars have shown that $\mathrm{CP}$ is not necessarily an automatic by-product of the conditional structure, even in the most archetypal examples such as (20) (see e.g. von Fintel 2001, van CanagemArdijns and van Belle 2008, Elder 2014).

If you mow the lawn I'll give you five dollars. (Geis and Zwicky 1975) 
As CP is the inference that $p$ describes the unique situation in which $q$ is expected to arise, it follows that for (20), CP would not arise when there are other ways of earning five dollars in addition to $p$.

The non-obtaining of CP for a given conditional can be explained by drawing insight from alternative semantics, assuming Rooth's (1996: 271) claim that the "position of focus in an answer corresponds with the questioned position in wh-questions". On the one hand, conditionals answering a question about $p$ (e.g. 'what happens if $p$ ?') put focus on the consequent, and in turn trigger an exhaustive list of consequents with respect to $p$. Then, the question of 'what happens if not- $p$ ?' is neither specified nor relevant to the exchange, and hence CP would not be expected to arise. On the other hand, in the contrasting case, Rooth's focus assumption would predict that conditionals answering a question about the consequent ('when does $q$ occur?') would put focus on the antecedent, then would trigger an exhaustive list of antecedents, and would thus give us CP. However, taking up Lilje's (1972: 540) observation that, "[(21)] could well be the first item on a list of responses to the question, 'How can I earn five dollars?'”, i.e. a question about the consequent, von Fintel (2001) notes that if one item is sufficient for the exchange, there is no need for the speaker to continue the list. Exhaustivity of answers is not necessary, as a partial answer to the question under discussion is sufficient. ${ }^{16}$ In other words, even when there is focus on the antecedent, $\mathrm{CP}$ is not a default inference, as other (often covert) antecedents are available in the context that would equally answer the question 'when does $q$ occur?'.

We can thus see why CP does not obtain in (18) and (19). In the case of 'if his hip gives way, I'm covered to drive', the question under discussion is with respect to $q$, namely, 'under what circumstances am I covered to drive?'. It is clear from the context that the one antecedent provided is not a sufficient condition for $q$ and that there are other antecedents that could satisfy the implicit question. Hence, CP does not arise as $p$ is simply offered as an example of when $q$ obtains. In other words, the coherence link of exemplification that indicates how to complete the incomplete conditional also restricts $\mathrm{CP}$, as there is no expectation that $p$ exhausts the situations where $q$ arises. So, (18) and (19) can both communicate conditional propositions as well as obtain their completions via the coherence link of elaboration or exemplification.

Overall, for the class of incomplete conditionals to which (18)-(19) belong, the completion is not directly copy-pasted from the previous discourse and, as a result, does not have a determinate syntactic structure; therefore, an account of syntactic ellipsis would not be appropriate. However, rather than resorting to pragmatic processes of a wider scope, we have shown here that the communicated completion is recoverable on the basis of a coherence link between the subsentence and the utterances that precede it.

\section{$\underline{4.3 \text { Incomplete conditionals with pragmatically recoverable completions }}$}

\footnotetext{
${ }^{16}$ Elder (2014) provides further examples of when and how this generalisation can be overridden, but such considerations go beyond the scope of this paper.
} 
The final cases that we consider are at the pragmatic end of the continuum of our classification, in which the contribution of syntactic information to the recoverable meaning is smaller while that of contextual, pragmatic information is greater. It is at this end that we place conditionals whose missing consequent does not correspond to a unique syntactic structure, nor to a unique coherence link. Instead, cases in this class in a sense give rise to a set of 'equivalent' and 'interchangeable' consequents insofar as all possible completions achieve the same discourse effects, without these effects being tied to a unique syntactic structure or even unique proposition. To illustrate what we mean by 'equivalent' and ‘interchangeable’ effects, let us consider (21), adapted from Haugh (2010: 362).

(21) B: And normally if you have lunch and then you go back after lunch, everyone finds this I think, that you're too...

A: That you're sleepy.

B: Yeah yeah, you can't concentrate $[\ldots]$.

(ICE-AUS, S1A-022: 197-200)

In (21), it is not clear whether B would have completed the conditional in his/her first utterance using exactly the same syntactic structure that A provided in their response. However, as B's subsequent response 'yeah yeah' indicates, (s)he accepts A's offered completion on the grounds that A and B's respective completions (among others) could be considered interchangeable. At the same time, note that it is not the case that any completion of the incomplete conditional would do, as B's initial utterance provides contextual constraints on admissible completions. What is not clear is the specific syntactic structure that speaker B would have used to express the intended message. At the linguistic level, 'You're sleepy' and 'You can't concentrate' are two different sentences expressing different propositions. Yet, conceptualising conversations in terms of series of 'moves in a language game' (cf. Lewis 1979), both sentences arguably offer an equivalent contribution at the level of discourse: they perform the move of describing the decrease in one's productivity after lunch. It is with this sense of 'equivalent' and 'interchangeable' that we argue that cases at the pragmatic end of the continuum of subsentential speech can take on board any completion from a contextually constrained set of appropriate completions, while still being susceptible to a semantic analysis at the conceptual level. Put differently, equivalent or interchangeable propositions are those that have an overlapping set of pragmatic implications, in that they would all achieve the same effects in the context of a particular conversation; no matter which specific proposition from this set is chosen, the communicative outcome would remain the same. ${ }^{17}$

We add to (21) our own observations from the ICE-GB, exemplified in (4), repeated below as (22), and (23)-(24).

(Casual conversation about common friends)

\footnotetext{
17 The idea of 'interchangeable propositions' is related to Sperber and Wilson's (1986: 228) notion of 'interpretative resemblance': the idea that two representations interpretively resemble one another when they share their contextual implications in a particular context. Thank you to an anonymous reviewer for drawing our attention to this comparison.
} 
A: And Karen and Ian want to buy her half of the mortgage out, so they'll have too much mortgage.

B: Yeah... It really is...

A: I know. With Ian only a tennis coach.

B: Well even now. I mean, if he has good rates, good bank rates, and he's got a steady job...

A: That's true.

(ICE-GB, S1A-036: 035)

(23) (Casual conversation about operations)

A: But a friend of mine pulled some cartilage in her leg when she was playing squash, and she had that done under local. And it was awful because they had to give her uh uh uh injection in her back. It was apparently really dangerous because if they get the wrong point there...

(ICE-GB, S1A-046: 064)

(24) (Casual conversation about religion)

A: I always wonder whether people are better off with faith or without, or, well, with faith, whether they've got something that I haven't. I don't think so, but I just wonder, you know, if they're happier.

B: They'd have to be... It really depends on the personality because it...

A: They need something to believe in.

B: What was that I heard about... I mean, if you have inner strength to cope with and the way you cope is basically you're coping with your external pressures... Now if you haven't got the inner strength to do that, sometimes you need help and I think a lot of people turn to that.

(ICE-GB, S1A-084: 151)

In (22), pragmatic inferencing based on the context and on world knowledge give rise to a consequent along the lines of 'A mortgage could be possible'. In (23), via similar pragmatic means we retrieve a consequent along the lines of 'There could be negative effects'. Finally, in (24), we recover a consequent along the lines of 'You can survive without faith'.

Importantly, in all of these cases the recoverable consequent does not correspond to a unique syntactic structure as pragmatically compatible contributions could be phrased in a number of different ways. For example, for (23), alternative linguistic structures could be more specific about what these negative effects could be, such as, 'She could be left paralysed'; similarly for (24) a structure with the same implications for the context at hand could be, 'You don't need religion to help you cope'. Yet, the pragmatically recoverable consequents belong to a set of compatible alternatives, suitably constrained for the purposes at hand. All such alternative consequents are expected to perform the same 'move in the language game' that is played in the given conversation: expressing that affording the mortgage is possible in (22), that negative effects could be caused if the surgery goes wrong in (23), and that one can cope without faith in (24).

Such examples would be excluded from syntactically oriented accounts such as those discussed in Section 3, on the grounds that they lack a determinate completion. Specifically, Stanley (2000: 408-409) argues that the lack of determinate linguistic structure means that 
such subsentences fail to make an assertion with determinate content. However, our examples (22)-(24) undeniably make meaningful contributions to their respective discourses. It is on this basis that we contend that, so long as such utterances have a recoverable consequent, irrespective of how determinate in structure that consequent is, they ought to be accounted for in a theory of meaning. Put simply, so long as they are meaningful in the sense that they give rise to truth-conditional propositions, they are susceptible to a semantic analysis.

At this point, the question arises of whether we could apply a truth-conditional semantic account to conditionals without syntactically determinate consequents. To address this, we need to reiterate that it is not the case that any consequent is admissible. Take, for example (23): given how well-defined the context is and how clear the direction of the contribution performed with the incomplete conditional is, it would be highly implausible that the speaker would go on and utter (23') following the if-clause without being considered uncooperative.

(23') It's unlikely that permanent harm could be caused.

This difficulty in cancelling the retrievable consequent in (23) - where the consequent pertains to a set of interchangeable yet equivalent contributions at the level of thought makes it viable as a strongly intended message. However, this message does not emerge solely out of the choice of words used; it emerges out of the interaction between those words and other contextual, extra-linguistic information. Thus, denying that a strongly intended meaning is available is counterintuitive, but aiming to give a syntactic representation for it is not the right way forward exactly because the recoverable consequent does not correspond to a unique linguistic structure.

It seems that in order to account for the entire class of incomplete conditionals, the truth-conditional unit needs to move beyond the syntactic structure, and to make room for pragmatic information. The advantage of truth-conditional representations that allow pragmatic considerations is that we can dispense with the need to choose a unique syntactic structure when representing the most strongly intended meaning of an utterance that cannot (and should not) be completed syntactically. At the same time, it needs to be emphasised that the salient pragmatically retrievable meanings of the cases at the pragmatic end of our continuum must have - and do have - constraints. These constraints at play are not merely linguistic in nature, but concern the discursive effect that the utterance achieves in the context at hand. Thus, the next question we address is: how do we know which consequents are members of the set of 'equivalent' and 'interchangeable' alternatives? To answer this question, we employ Stalnaker's $(1978,2014)$ idea of context as common ground that is built from information that accumulates between interlocutors as conversation progresses. This common ground makes up a set of possibilities shared between participants, i.e. the set of possible ways in which the world could be that are compatible with the information available in the common ground. On that view, the proposition communicated with each new utterance is seen as a proposal to update the common ground by providing information regarding the available possibilities; that is, by verifying some of them as actual, while ruling others out as non-actual. This common ground then also renders certain possibilities compatible, while ruling others out as incompatible. Seen in this way, the context in which (23) was uttered will include information regarding the difficulty or level of expertise required for the relevant medical operations. Uttered against this background of information, the context in which the 
incomplete conditional in (23) was uttered rules out any proposition that is incompatible with the new information offered by the incomplete conditional itself, e.g. that the risk of the operation is low. At the same time, it leaves open a relatively constrained set of consequents that communicate the message that negative effects could be caused if the surgery does not go well, e.g. that the speaker's friend could be left paralysed, or that some other serious harm could be caused. ${ }^{18}$

To sum up, the motivation behind placing cases such as (22)-(24) at the pragmatic end of the continuum of incomplete conditionals is that their context of utterance is precise enough to safeguard the meaningfulness of such conditional utterances despite the syntactic indeterminacy of the consequents they give rise to. Put differently, because the contextual contribution in these cases is greater, the contribution of linguistic information can be kept minimal. The idea then is that an incomplete conditional uttered in an informationally rich context can achieve the same effect that a complete conditional would achieve in an informationally poorer context. In other words, not only is the recovery of a consequent of an incomplete conditional constrained by the information included in the shared background, but whether the use of an incomplete conditional in place of a complete one is appropriate will also depend on the information available and salient in the common ground.

\section{Concluding remarks}

In this paper, we have assumed that it is uncontroversial that incomplete conditionals of the form 'if $p$ ' are, indeed, conditionals that communicate propositions. We have seen that the ways in which the consequent can be recovered are context-dependent, and the extent to which a syntactically or pragmatically oriented solution is required depends on the availability of a salient, structurally determinate consequent in the prior discourse. To summarise, in cases where a syntactically determinate consequent is not available but the conditional appears conceptually complete, pragmatics is expected to play a more substantive role in the recovery of the intended interpretation and vice versa. We thus proposed that the instances of incomplete conditionals lie on a continuum, as illustrated in Figure 1.

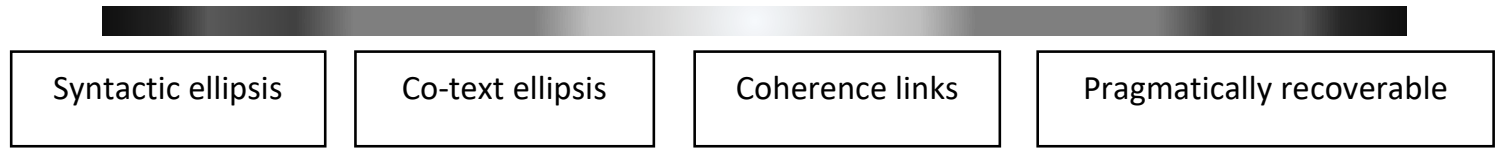

Figure 1: The continuum of incomplete conditionals (adapted from Savva 2017)

While the categories discussed in this paper imply discrete boundaries between the cases, the notion of the continuum is supposed to reflect the fact that both syntactic and pragmatic

\footnotetext{
${ }^{18}$ See Savva (2017) for an application of this account of indeterminacy to cases of fragments beyond incomplete conditionals.
} 
information are involved in all cases to different extents. As such, it is worth emphasising that differences between the categories is gradient rather than strict, and that our classification is indicative of those differences but not necessarily exhaustive of the ways in which syntax and pragmatics can be used to recover an implicitly communicated consequent. But most importantly, all cases represented on the continuum communicate intuitively truth-conditional content, and hence ought to be susceptible to a semantic analysis.

This brings us to the broader question over the appropriate unit of semantic analysis on the assumption that semantic theory aims to derive intuitive truth-conditional propositions - which speaks to the division of labour between semantics and pragmatics in deriving propositional forms. If we were to take a strict view on the extent to which context is allowed to intrude on the unit of semantic analysis, following e.g. Stanley's (2000) indexicalism detailed in Section 3, we would only be allowed to look at elements in the structure to inform our propositions. However, we have shown that we sometimes need to allow pragmatics to play a greater role in obtaining the propositional forms that align with speakers' intuitions regarding truth conditions. To that end, we agree with Recanati's (2010) contextualist view of semantics which allows elements in the syntactic structure to be pragmatically enriched or modified. At the same time, we also agree with Recanati that even pragmatically retrievable interpretations are - to some extent - grammatically constrained; in the case of incomplete conditionals, these constraints are given by the 'if $p$ ' form which signals that there is a $q$ to be recovered. So, in the spirit of Vicente and Groefsema (2013), it seems that a satisfactory account of linguistically incomplete sentences would be better off if it viewed the contribution of syntax as placing constraints on what the completion could be, rather than as being solely responsible for providing the completion itself across the board.

Overall, the case of incomplete conditionals has shown us that the unit of semantic analysis has to be broadened beyond that of overtly pronounced sentential structures and that pragmatic inferencing has to be allowed to do much of the work in deriving propositional content. Simply taking a sentence-based view, or even an uttered sentence-based view, where sentences pertain to fully sentential structures, would leave subsentential utterances, including incomplete conditionals, out of its scope. However, as we have shown, in ordinary discourse incomplete conditionals can be used to express meaningful, propositional forms in context. In fact, from the pragmatic view on conditionals that we employ, we go so far as to say that the completion of an implicit consequent is necessary to make sense of the whole thought that is communicated via an incomplete conditional. But while the completion of a consequent is necessary to obtain a conditional thought, it is not necessarily contributed by syntax. At the same time, even in the most pragmatic cases (see Section 4.3), the sentence fragment still has a role to play in the formulation of truth-conditional content as the antecedent $p$ signals that a conditional thought is being entertained. Thus, although there are no truly syntax-only solutions for recovering the consequent of an incomplete conditional, syntax nevertheless plays a crucial role: syntax signals that something is missing, but very often it is pragmatics that tells us what it is.

\section{$\underline{\text { References }}$}

Adams, E. W. 1975. The Logic of Conditionals: An Application of Probability to Deductive Logic. Dordrecht: D. Reidel. 
Asher, N. and A. Lascarides. 2003. Logics of Conversation. Cambridge: Cambridge University Press.

Atlas, J. D. and S. C. Levinson. 1981. 'It-clefts, infomativeness and logical form: Radical pragmatics'. In P. Cole (ed.). Radical Pragmatics. New York: Academic Press. 1-61.

Austin, J. L. 1961. 'Ifs and cans'. In J. O. Urmson and G. J. Warnock (eds.). Philosophical Papers. Oxford: Oxford University Press. 153-180.

Van der Auwera, J. 1997. 'Pragmatics in the last quarter century: The case of conditional perfection'. Journal of Pragmatics 27. 261-274.

Bach, K. 1994. 'Semantic slack: What is said and more'. In S. L. Tsochatzidis (ed.). Foundations of Speech Act Theory. Philosophical and Linguistic Perspectives. London, New York: Routlege. 267-291.

Bach, K. 1999. 'The myth of conversational implicature'. Linguistics and Philosophy 22. 327-366.

Bach, K. 2007. 'Regressions in pragmatics (and semantics)'. In N. Burton-Roberts (ed.). Pragmatics. Basingstoke: Palgrave Macmillan. 24-44.

Barton, E. 1990. Nonsentential Constituents: A Theory of Grammatical Structure and Pragmatic Interpretation. Amsterdam: John Benjamins.

Bezuidenhout, A. 2006. 'VP-ellipsis and the case for representationalism in semantics'. ProtoSociology 22. 140-169.

Borg, E. 2012. Pursuing Meaning. Oxford: Oxford University Press.

Van Canegem-Ardigns, I. and W. Van Belle. 2008. 'Conditionals and types of conditional perfection'. Journal of Pragmatics 40. 349-376.

Cann, R., R. M. Kempson and L. Marten. 2005. The Dynamics of Language: An Introduction. Amsterdam: Elsevier.

Carston, R. 2002. Thoughts and Utterances. Oxford: Blackwell. 
Cinque, G. and L. Rizzi. 2010. 'The cartography of syntactic structures'. In B. Heine and N. Heiko (eds). The Oxford Handbook of Linguistic Analysis. Oxford: Oxford University Press. 65-78.

Clapp, L. 2005. 'On the interpretation and performance of non-sentential assertions'. In R. Elugardo and R. J. Stainton (eds.). Ellipsis and Non-Sentential Speech. Dordrecht: Springer. 109-129.

Clapp, L. 2012. 'Three challenges for indexicalism'. Mind and Language 27. 435-465.

van Craenenbroeck, J. and J. Merchant. 2013. 'Ellipsis phenomena'. In M. den Dikken (ed.). The Cambridge Handbook of Generative Syntax. Cambridge: Cambridge University Press. 701-745

Edgington, D. 1986. 'Do conditionals have truth conditions?'. Crítica: Revista Hispanoamericana de Filosofía 18. 3-39.

Elder, C. 2014. On the forms of conditionals and the functions of 'if'. PhD thesis, University of Cambridge.

Elder, C. and D. Beaver. 2017. 'The optimal rate of miscommunication'. Paper presented at Communication \& Cognition 2017 (ComCog), University of Fribourg.

Elder, C. and K. M. Jaszczolt. 2016. 'Towards a pragmatic category of conditionals'. Journal of Pragmatics 98. 36-53.

Elugardo, R. and R. J. Stainton. 2005. 'Introduction'. In R. Elugardo and R. J. Stainton (eds.). Ellipsis and Non-Sentential Speech. Dordrecht: Springer. 1-26.

Evans, N. 2007. 'Insubordination and its uses'. In I. Nikolaeva (ed.). Finiteness: Theoretical and Empirical Foundations. Oxford: Oxford University Press. 366-431.

Von Fintel, K. 2001. 'Conditional strengthening'. Unpublished manuscript.

Ford, C. E. and Thompson, S. A. 1986. 'Conditionals in discourse: A text-based study from English'. In E. C. Traugott, A. T. Meulen, J. S. Reilly and C. A. Ferguson (eds.). On Conditionals. Cambridge: Cambridge University Press. 353-372.

Franke, Michael. 2009. Signal to act: Game theory in pragmatics. Amsterdam: Institute for Logic, Language and Computation (ILLC). PhD thesis. 
Geis, M. L. and A. M. Zwicky. 1971. 'On invited inferences'. Linguistic Inquiry 2. 561-566.

Grice, H. P. 1967. 'Indicative conditionals'. Reprinted in H. P. Grice 1989. Studies in the Way of Words. Cambridge, MA: Harvard University Press. 22-40.

Grice, H. P. 1989. Studies in the Way of Words. Cambridge, MA: Harvard University Press.

Hale, J. 2011. 'What a rational parser would do'. Cognitive Science 35. 399-443.

Hall, A. 2009. 'Subsentential utterances, ellipsis, and pragmatic enrichment'. Pragmatics and Cognition 17. 222-250.

Haugh, M. 2010. 'Co-constructing what is said in interaction'. In T. E. Németh and K. Bibok (eds.). The Role of Data at the Semantics/Pragmatics Interface. Berlin: Mouton de Gruyter. 349-380.

Horn, L. 2000. 'From if to iff: Conditional perfection as pragmatic strengthening'. Journal of Pragmatics 32. 289-326.

Jaszczolt, K. M. 2016. Meaning in Linguistic Interaction: Semantics, Metasemantics, Philosophy of Language. Oxford: Oxford University Press.

Kamp, H. and U. Reyle. 1993. From Discourse to Logic. Kluwer: Dordrecht.

Kehler, A. 2002. Coherence, Reference, and the Theory of Grammar. Stanford, CA: CSLI Publications.

Levinson, S. C. 1983. Pragmatics. Cambridge: Cambridge University Press.

Lewis, D. 1979. 'Scorekeeping in a language game'. Journal of Philosophical Logic 8. 339359.

Lilje, G. W. 1972. 'Uninvited inferences'. Linguistic Inquiry 3. 540-542.

Ludlow, P. 2005. 'A note on alleged cases of nonsentential assertion'. In R. Elugardo and R. J. Stainton (eds.). Ellipsis and Non-Sentential Speech. Dordrecht: Springer. 95-108.

Martí, L. 2015. 'Grammar versus pragmatics: Carving nature at the joints'. Mind and Language 30. 437-473.

Merchant, J. 2004. 'Fragments and ellipsis'. Linguistics and Philosophy 27. 661-738. 
Merchant, J. 2006. 'Small structures: A sententialist perspective'. In L. Progovac, K. Paesani, E. Casielles and E. Barton (eds.). The Syntax of Nonsententials: Multidisciplinary Perspectives. Amsterdam, Philadelphia: John Benjamins. 73-91.

Merchant, J. 2010. 'Three kinds of ellipsis: Syntactic, semantic, pragmatic?'. In F. Recanati, I. Stojanovic and N. Villanueva (eds.). Context-Dependence, Perspective, and Relativity. Berlin: Mouton de Gruyter. 141-192.

Neale, S. 1990. Descriptions. Cambridge, MA: MIT Press Books.

Neale, S. 2004. 'This, That and the Other'. In M. Reimer and A. Bezuidenhout (eds.). Descriptions and Beyond: An Interdisciplinary Collection of Essays on Definite and Indefinite Descriptions. Oxford: Oxford University Press. 68-182.

Quirk, R., S. Greenbaum, G. Leech and J. Svartvik. 1985. A Comprehensive Grammar of the English Language. London, New York: Longman.

Ramsey, F. P. 1929. 'General propositions and causality'. Reprinted in D. H. Mellor (ed.) 1990. Philosophical Papers. Cambridge: Cambridge University Press. 145-163.

Recanati, F. 2004. Literal Meaning. Cambridge: Cambridge University Press.

Recanati, F. 2010. Truth-Conditional Pragmatics. Oxford: Clarendon Press.

Rooth, M. 2006. 'Focus'. In S. Lappin (ed.). The Handbook of Contemporary Semantic Theory. Oxford: Blackwell. 271-298.

Savva, E. 2017. Subsentential Speech from a Contextualist Perspective. PhD thesis, University of Cambridge.

Stainton, R. J. 2006. Words and Thoughts: Subsentences, Ellipsis and the Philosophy of Language. Oxford: Oxford University Press.

Stalnaker, R. C. 1975. 'Indicative conditionals'. Reprinted in R. C. Stalnaker 1999. Context and Content: Essays on Intentionality in Speech and Thought. Oxford: Oxford University Press. 63-77.

Stalnaker, R. C. 1978. 'Assertion'. Reprinted in R. C. Stalnaker 1999. Context and Content: Essays on Intentionality in Speech and Thought. Oxford: Oxford University Press. 78-95.

Stalnaker, R. C. 2014. Context. Oxford: Oxford University Press. 
Stanley, J. 2000. 'Context and logical form'. Linguistics and Philosophy 23. 391-434.

Stanley, J. 2007. Language in Context: Selected Essays. Oxford: Oxford University Press.

Stirling, L. 1999. 'Isolated if-clauses in Australian English'. In P. Collins and D. Lee (eds.). The Clause in English. Amsterdam, Philadelphia: John Benjamins. 273-294.

Vallauri, E. L. 2004. 'Grammaticalization of syntactic incompleteness: Free conditionals in Italian and other languages'. SKY Journal of Linguistics 17. 189-216.

Vicente, B. and M. Groefsema. 2013. 'Something out of nothing? Rethinking unarticulated constituents'. Journal of Pragmatics 47. 108-127.

Xiang, M.; J. Grove; J. Merchant. 2014. 'Ellipsis sites induce structural priming effects'. Manuscript. University of Chicago.

Yanofsky, N. 1978. 'NP utterances'. Chicago Linguistics Society 14. 491-502. 